Check for updates

Cite this: RSC Adv., 2017, 7, 25657

\title{
Phase-conjugated directional diffraction from a retroreflector array hologram
}

\author{
Rajib Ahmed, (D) *a Ahmmed. A. Rifat, ${ }^{\text {b }}$ Muhammad Umair Hassan, ${ }^{\text {ac }}$ Ali K. Yetisen (D) d \\ and Haider Butt (D)*a
}

A corner cube retroreflector (CCR) consists of three perpendicular flat surfaces and reflects the incident light back to its source. Optical properties of CCR arrays have applications in free space optical communication, low power wireless networks and sensing applications. Conventional top-down CCR array fabrication is complex and requires expensive equipment and limited to broadband reflection only. Here, we utilize laser assisted copying of a CCR array to a light sensitive holography polymer film $(\sim 10 \mu \mathrm{m})$ which was placed parallel to the object plane (CCR array) during the recording. Optical characterization of the recorded CCR array hologram was carried out using reflection and color-selective diffraction measurements. Angle dependent optical properties were also simulated computationally followed by their experimental realization, which confirm our experimental findings. In a broadband illumination setting, a broadband white light reflection and a narrowband color diffraction were observed. A linear relationship between the incidence angle of the broadband light and the diffraction angle of the diffracted color light was observed. Bright and well-defined $2^{\text {nd }}$ order far-field diffraction patterns were observed using an image-screen experiment. Maximum diffraction efficiency (DE) of $\sim 50 \%$ was observed for the monochromatic green light at normal illumination setting. The farfield diffraction interspacing/intensity exhibits increasing/decreasing trend with illumination tilt angles, measured between 10 to 50 degrees. The recorded CCR array holograms offer potential applications in color selective diffraction optics and customized optical devices.

Received 11th April 2017

Accepted 2nd May 2017

DOI: $10.1039 / \mathrm{c7ra04131d}$

rsc.li/rsc-advances
CCRs including microelectromechanical, lithography, and direct laser writing. ${ }^{13-15}$ Diamond micro-maching was also used to directly cut $120^{\circ} \mathrm{CCR}$ faces for high retroreflection. ${ }^{16} \mathrm{CCRs}$ have also been fabricated by direct etching using different masks obtained by photolithography. ${ }^{14,17}$ Direct laser ablation technique has also been used for the same purpose. ${ }^{18}$ However, these reported techniques have limitations due to relatively expensive equipment, time-consuming processing and high expertise-dependence. ${ }^{14,19,20}$ Therefore, fast, low cost, and accurate techniques are desired for CCRs manufacturing. Holography technique with desired surface functionalities have been recently reported to produce CCRs and other optical device (lens, diffuser, 1D/2D patterns, and diffraction grating, etc.), which is a simple, fast, and cost-effective process. ${ }^{21-23}$

Holography is mainly classified into two types, surface or two-dimensional (2D) holography and volume or threedimensional (3D) holography. ${ }^{24}$ Both technique are based on optical recoding of phase and amplitude information of reflected light from a recorded object. ${ }^{25}$ An optical recording medium (ORM) is a photosensitive material, usually a polymer, that retains the optical information it has been exposed to. Recording on ORM for a 3D hologram is realized by illuminating it with two interfering beams coming from the same source but different pathways, that is, a reflection beam from
${ }^{a}$ Nanotechnology Laboratory, School of Engineering, University of Birmingham, Birmingham B15 2TT, UK. E-mail: a.rajib@osamember.org; h.butt@bham.ac.uk

${ }^{b}$ Nonlinear Physics Centre, Research School of Physics \& Engineering, Australian National University, Canberra, ACT-2601, Australia

'Department of Physics, COMSATS Institute of Information Technology, Islamabad 44000, Pakistan

${ }^{d}$ Harvard-MIT Division of Health Sciences and Technology, Massachusetts Institute of Technology, Cambridge, MA, 02139, USA 
the object, and a guided reference beam from the source. After the recording process, image reconstruction is achieved through light reflection/diffraction from the recorded information on the ORM. In 2D-holography, light information from both reference and recording medium comes from the same side and creates perpendicular planar interference fringes on the ORM. ${ }^{24}$ However, in 3D-holography, light information coming from different sides of the reference and recoding of medium make multilayer fringes on the ORM photo-polymers. With the variation of input light intensity, wavelength, phase and physical arrangements, micro and nano scale 2D/3D optical devices can be recoded thorough reflection holography. ${ }^{22}$ Holographic recording through a light sensitive medium has applications in displays, optical devices, sensing, interferometry, nano-antennas and data storage. ${ }^{26,27}$

Here, a CCR array hologram is recorded on a light sensitive ORM through Denisyuk reflection holography method. Optical properties of the recorded hologram were studied using reflection, diffraction, rational, and far-field experiments, as well as, by computational modelling. Far-field experiments showed bright and well-resolved $2^{\text {nd }}$ order diffraction patterns with monochromatic light (red, green, and blue) illuminations. As the incident wavelength increased, the interspacing between diffraction spots and non-diffracted zero order also increased. The diffraction intensity decreased as the incident light wavelength decreased, in the same way as expected from a diffraction grating. The DE measurement exhibited a maximum of $\sim 50 \%$ (minimum of $\sim 18 \%$ ) light diffraction for the green (blue) illuminations. The CCR array hologram showed angular dependence of white light reflection and exhibited a color resolved diffraction when subject to a broadband illumination. Angle dependent behavior of the recorded hologram was also confirmed by computational simulations for triangular array structures. Image projection experiments were also carried out to study the rotational property of the CCR array hologram.

\section{Result and discussion}

\section{Fabrication and sample preparation}

Silver-halide chemistry was used for the preparation of the holographic plate samples, see Fig. 2. An aqueous solution of 30 $\mathrm{mL}$ triethanolamine (TEA) $(1: 300, \mathrm{v} / \mathrm{v})$ was used as wetting agent (mixed and stirring for 2 to $3 \mathrm{~min}$ ). Holographic plates of $20 \times 20 \mathrm{~cm}^{2}$ (BB-640, Colour Holographic, London) were soaked into this solution ( 2 to $3 \mathrm{~min}$ ) for pre-swilling to achieve higher light-sensitivity and color shifting property of the recorded hologram. The holographic emulsion medium was based on thin $(\sim 10 \mu \mathrm{m})$ gelatin layers which contains AgBr nano-crystals (NCs) that act as the light sensitive medium. Subsequently, the pre-swelled plates were dried under laminar air flow. These plates (one at a time) were then placed on a laser exposure stage for the recoding. We used a Denisyuk reflection holography sachem, as this is the simplest technique, yet, powerful enough to produce highest quality holograms. An expanded continuous red laser (632.13 nm, $4 \mathrm{~mW}$ ) was use to expose (at normal angle) the holographic plate (sample) and the CCR array (object) (SL150-18, JunAN, China) was placed - at distance of $40 \mathrm{~cm}$ in a top-bottom configuration, respectively.

After passing through the optical components, the directed light passes through the ORM and reflects back from the object to the ORM where it leaves interference imprints - thus, the information is recorded. The recoding process required only few seconds to record the CCR hologram array. The latent image was formed through redistribution of $\mathrm{AgBr}$ NCs in two steps, namely, ${ }^{28}$ ionization: $\mathrm{AgBr}+h \nu \rightarrow \mathrm{Ag}^{+}+\mathrm{Br}^{-}+\mathrm{e}^{-}$, and metallic $\mathrm{Ag}^{0}$ formation: $\mathrm{Ag}^{+}+\mathrm{e}^{-} \rightarrow \mathrm{Ag}^{0}$. The recorded hologram was

(a)

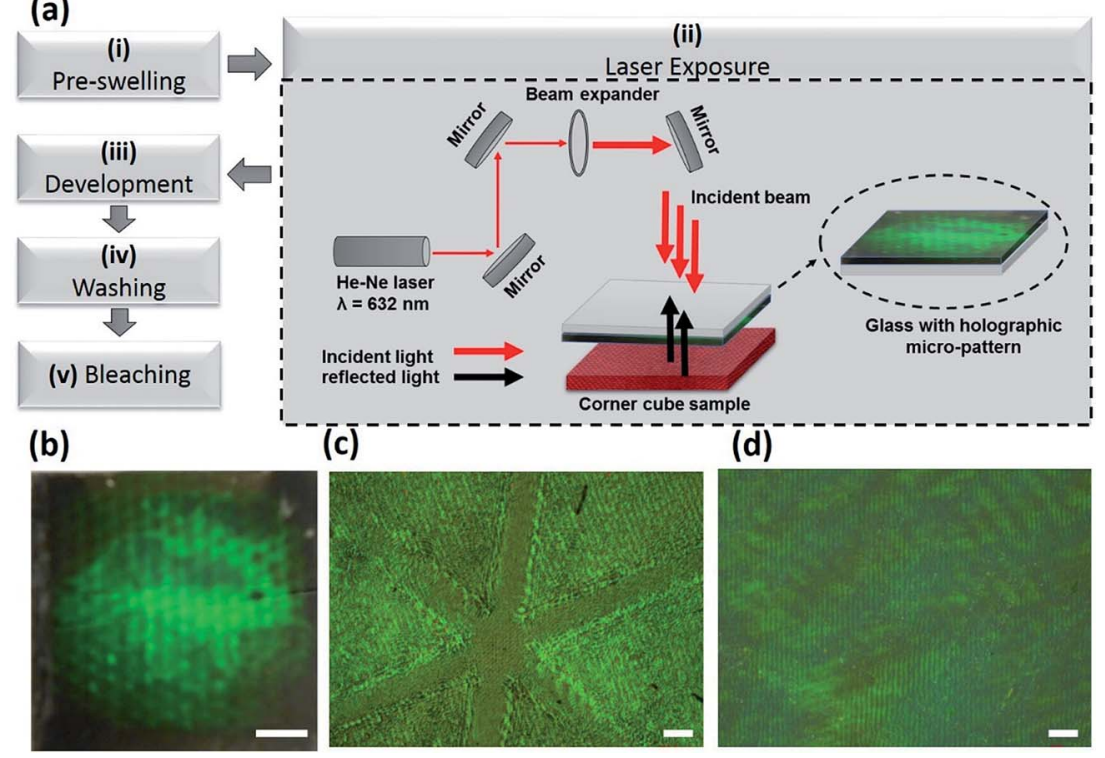

Fig. 1 (a) Steps involved in sample preparation of CCR array hologram (i-v), the schematic of experimental setup used for optical recoding of CCR array hologram (dotted gray box) as step (ii). (b-d) Photograph and magnified microscopic images of CCR array hologram showing triangular section, and internal structure of the recorded hologram. Scale bars $=3 \mathrm{~cm}, 200$, and $20 \mu \mathrm{m}$. 


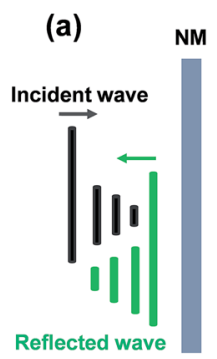

(b)

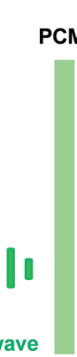

(c)

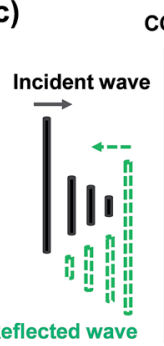

(d)

(e)

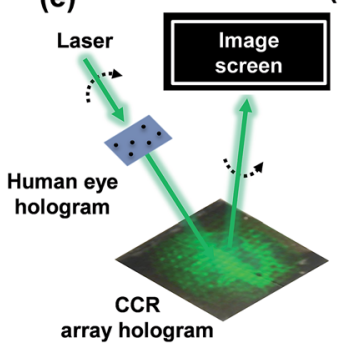

(f)

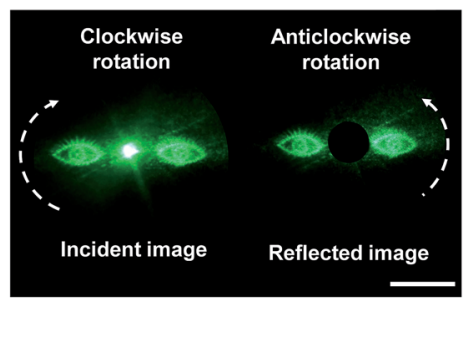

Fig. 2 Optical property of NM, PCM, and CCR array. (a-c) The incident and reflected light wave from NM, PCM, and CCR array. (d) Triangular grating structure of the CCR array. (e) Experiment setup for the phaseconjugated rotational property through image projection experiment. (f) Incident image on CCR array hologram and reflected image on an image screen setup.

immersed (5 to $6 \mathrm{~min}$ ) in a developer based on equal amounts of solution-A (ascorbic acid (40 gm), 4-methylaminophenol (6 gm) in $1 \mathrm{~L}$ DI-water), and solution-B (sodium carbonate (100 gm), sodium hydroxide $(30 \mathrm{gm})$ in $1 \mathrm{~L}$ DI-water. Formation of $\mathrm{Ag}^{0}$ nanoparticles was favored at points of bright fringes, and appear as dark image in the holographic plate. The recorded holographic plate was removed from the developer bath and immersed ( 1 to $2 \mathrm{~min}$ ) in the bleach containing potassium bromide $(\mathrm{KBr})(60 \mathrm{~g})$ and $\mathrm{CuSO}_{4}(30 \mathrm{~mL})$ solution. During the bleaching process, the dark image converted to the bright image. Finally, the recorded hologram was washed (1-2 min) in a water bath and the dried (1-2 min) using hot laminar flow hologram was ready for optical characterization. The whole process was performed in safety green light and completed in 20-30 minutes.

\section{Optical property of a retroreflector}

The CCR retroreflector consists of three trianglular mirror surfaces at right angles with two possible configurations, hollow, and solid CCR. ${ }^{3}$ A light beam incident to the CCR undergoes three mirror reflections before returning back to the source. However, not all the light entering from the front face of CCR experiences a complete reflection from all mirror surfaces - some of it becomes part of the retro-reflection. ${ }^{29}$ Notice that it is only the middle part of the triangular sections that plays active role in the retroreflection. ${ }^{4,30}$ Optical properties of the CCR are displayed through reflection and phase-conjugation rotational properties.

Suppose an incident plane wave under paraxial approximation, propagating along $x$-direction,

$$
E_{\mathrm{i}}(x, y, z)=A(x, y) \exp (i \varphi(x, y)) \exp (-i k z)
$$

From normal mirror (NM), the reflected light wave,

$$
E_{\mathrm{m}}(x, y, z)=A(x, y) \exp (i \varphi(x, y)) \exp (+i k z)
$$

For ideal phase conjugation mirror (PCM) case,

$$
E_{\mathrm{PC}}(x, y, z)=A(x, y) \exp (-i \varphi(x, y)) \exp (+i k z)
$$

In a large CCR array,

$$
\begin{aligned}
E_{\mathrm{CCR} \_a r r a y}(x, y, z)= & \sum_{m, n} A(2 m h-x, 2 n h-y) \\
& \times \exp (i \varphi(2 m h-x, 2 n h-y)) \exp (+i k z) a_{\mathrm{CC}} \\
& \times(x, y) \operatorname{rect}\left(\frac{x-m h}{h}, \frac{y-n h}{h}\right)
\end{aligned}
$$

where, $m, n$ represent each optical field segment reflected by each corner cube, $A(x, y), \phi(x, y)$ and $\operatorname{rect}(x, y)$ are amplitude, phase and rectangular functions, respectively. The rectangular function is given by

$$
\operatorname{rect}(x, y)=\left\{\begin{array}{lc}
1 \quad \text { if } \quad \operatorname{abs}(x, y)<\frac{1}{2} \\
0 \quad \text { if } \operatorname{abs}(x, y)>0
\end{array}\right.
$$

$E_{\mathrm{CCR} \_ \text {array }}$ can be realized as an ideal $E_{\mathrm{PC}}$ phase conjugation using eqn (3) and (4) considering,

(a) a constant amplitude approximation, that is, the amplitude variation should very small in each corner cube as if constant, therefore,

$A(x, y) \operatorname{rect}\left(\frac{x-m h}{h}, \frac{y-n h}{h}\right) \approx A(m x, n y) \operatorname{rect}\left(\frac{x-m h}{h}, \frac{y-n h}{h}\right)$

and, (b) a linear phase distribution inside CC area, such that,

$$
\begin{aligned}
\phi(x, y) \operatorname{rect}\left(\frac{x-m h}{h}, \frac{y-n h}{h}\right) \approx \\
\phi\left(k_{x, m, n} x+k_{y, m, n} y\right) \operatorname{rect}\left(\frac{x-m h}{h}, \frac{y-n h}{h}\right)
\end{aligned}
$$

As a result eqn (4) can be written as,

$$
\begin{aligned}
E_{\text {CCR_array }}(x, y, z)= & \sum_{m, n} A(m h, n h) \exp \left(i 2 m h k_{x, m, n}+i 2 n h k_{y, m, n} y\right) \\
& \times \exp (+i k z) a_{\mathrm{CC}}(x, y) \operatorname{rect}\left(\frac{x-m h}{h}, \frac{y-n h}{h}\right)
\end{aligned}
$$

where, $\exp \left(i k_{x, m, n} x-i k_{y, m, n} y\right)$ and $\exp \left(i 2 m h k_{x, m, n}+i 2 n h k_{y, m, n}\right)$ represent the phase conjugation and phase mismatch between the segments, respectively.

In terms of amplitude and phase reversal of the reflected wave, NM, PCM, and CCR hold different properties, see Fig. 2ac. The incident wave reflected from a NM is oriented normal to the direction of propagation. The reflected wavefront from PCM shows the phase reversal property in contrast with that reflected 
from a CCR or NM. CCR faces are triangular, projection (side view), which can be visualized as $2 \mathrm{D}$ triangular grating structures, see Fig. 2d. The wavefront reflected from these triangular grating structures is completely disrupted and each section of the wavefront is parallel to the PCM. Therefore, the CCR acts as a pseudophase phase conjugator compared with PCM by reversing all segments of the reflected wavefront. ${ }^{3}$ The recorded CCR hologram also showed similar pseudophase phase conjugation properties as probed by image projection and rotational experiments. A pair of human eye image was illuminated on the recorded CCR hologram and far-field image was captured using an imaging setup, see Fig. 2e. The far-field projected image from the CCR hologram surface rotates in the opposite direction to the incident image ('human eye') for a monochromatic (green) light source, see Fig. 2f. Phase-conjugated rotational properties are also valid for other monochromatic (red, blue) and broadband light illuminations.

\section{Optical computational modelling}

Optical reflection modelling of the CCR array was performed using finite element method (FEM). ${ }^{31,32}$ 2D simulation was performed in order to reduce the computational complexity and runtime. The triangular grating surface $(n=2.24$ at $632 \mathrm{~nm})$ was illuminated with a light source at its normal $\left(90^{\circ}\right)$ and tilted angles $\left(-30^{\circ}\right.$ and $\left.-60^{\circ}\right)$. The reflected light was measured from the hemispherical surface surrounded by air $(n=1)$. Continuity and scattering boundary conditions were considered at the triangular grating and hemispherical surface during simulation. Sub-meshing (one tenth of incident light) and convergence tests were performed for the accuracy of our results. ${ }^{33} \mathrm{Compu}-$ tation structure consisted of 14031 triangular mesh-elements, 647 boundary elements and 98836 degrees of freedom, solution time was $\sim 30$ s. Fig. 3a shows the conceptual block diagram for the computational reflection from the CCR surface. During normal reflection, source and analyzer were considered at the same position. Light reflected at normal possesses the maximum intensity of the reflected green color from the grating surface. The broadband light illumination at $-30^{\circ}$ and $-60^{\circ}$ tilt (a)
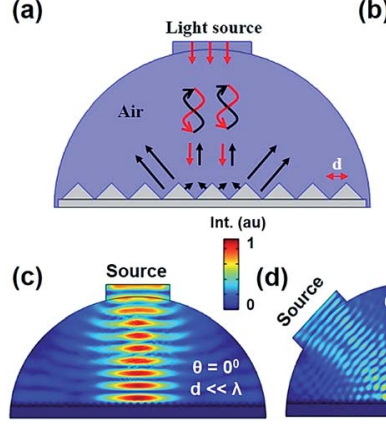

(d)

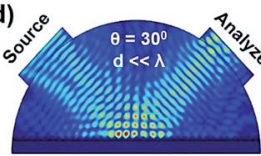

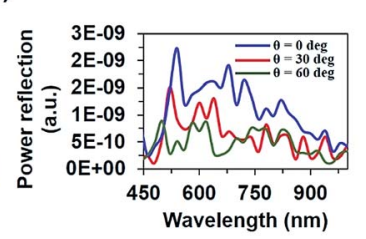

(e)

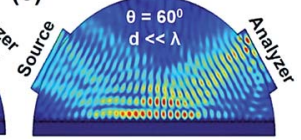

Fig. 3 Computational modelling of reflection property of CCR array (a) Conceptual simulation block diagram. (b) Reflected power intensity as a function wavelength for the illumination angle variation $(\theta=0$, $30^{\circ}$, and $\left.60^{\circ}\right)$. (c-e) Electric field intensity distribution of the normal $(\theta$ $=0$ ), and tilted $\left(\theta=0,30^{\circ}\right.$, and $\left.60^{\circ}\right)$ light illumination to rectangular CCR curved surface.

showed reflections at $+30^{\circ}$ and $+60^{\circ}$, respectively, as assessed by a spectrum analyzer computation module at these angles. The reflected light intensities plotted as a function of wavelength at illumination angles of $90^{\circ}, 30^{\circ}$ and $60^{\circ}$, showed peak maxima at 540, 520 and $500 \mathrm{~nm}$, respectively, see Fig. 3b. In the reflection spectra, random peaks also existed apart from the peak shifts. The overall reflected intensity was maximum for the normal illumination and reduced for higher angles. It was difficult to perform the computation for very large angle because of geometrical constraints.

The electric field distribution of the computational results with normal and tilted broadband light illumination exhibited reflection, interference, diffraction/scattering properties as shown in Fig. 3c-e. Interference patterns were observed from the periodic intensity variation along the vertical axis. For the normal illumination, maximum light was reflected back toward the source and minimum diffraction/scattering was observed. Inference occurs between the incident (red arrow) and reflected (black arrow) light waves. Light inference for $60^{\circ}$ tilted angle was higher as compared with the $30^{\circ}$ tilted angle. Interference intensity for the tilted illumination is also dependent on the number of side walls. For larger tilt angles, more light is reflected from the side walls and has more chance to interfere with the incident waves. Therefore the amount of light diffraction/scattering increased for the large tilt illumination $(\theta$ $\left.=60^{\circ}\right)$ compared with lower tilt angle $\left(\theta=30^{\circ}\right)$ and minimum for the normal illumination $\left(\theta=0^{\circ}\right)$.

\section{Optical characterization}

Experimental studies for optical properties of the CCR array hologram were carried out in reflection mode using a reflection/
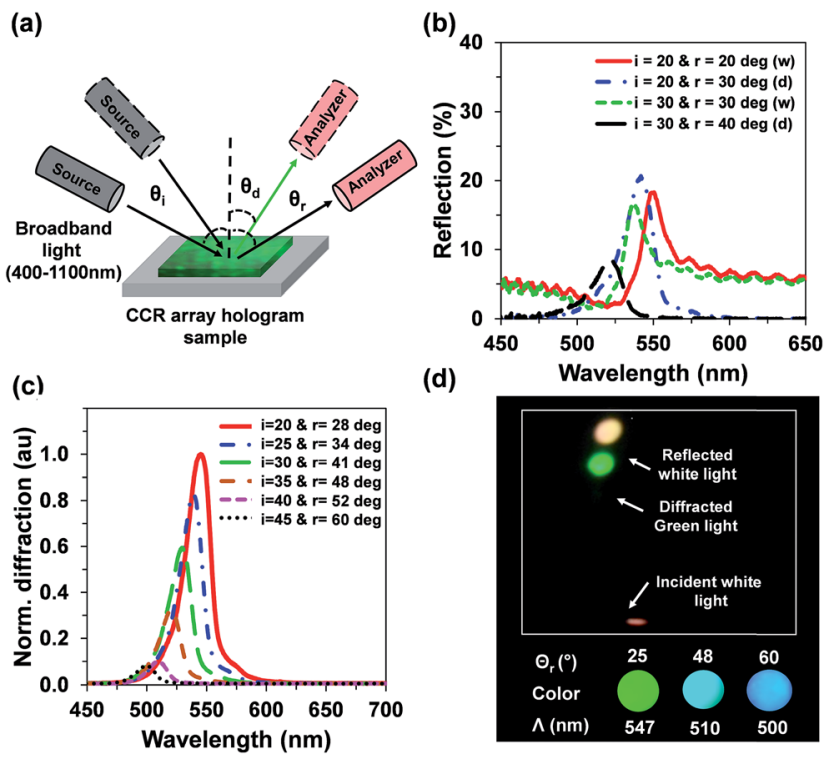

(d)

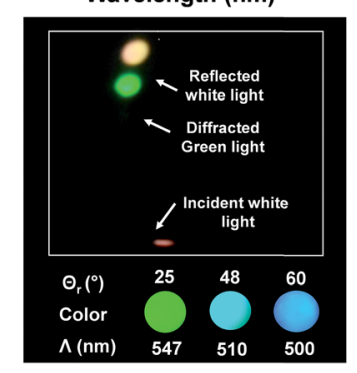

Fig. 4 Optical property of the CCR hologram. (a) Conceptual block diagram for the tilted reflection and diffraction measurements. (b) Tilted reflection as function of wavelength. (c) Normalized diffraction as a function of wavelength. (d) Far-field diffracted colors light on an image screen. 
diffraction measurement setup, see Fig. 4a for the details. The CCR array hologram surface was illuminated with a broadband light (400-1100 nm) at an incident angles, $\theta_{\mathrm{i}}$. Reflection and diffraction intensities were measured at angles $\theta_{\mathrm{r}}$, and $\theta_{\mathrm{d}}$, respectively, using a photospectrometer (Ocean Optics, $\Delta \lambda=$ 450-1100 nm, resolution $=0.2 \mathrm{~nm}$ ). The light source and spectrometer were carefully mounted on a precision motorized rotation stages for angular measurements. The setup can be said to consist on two main halves, one for incident light and other for reflection/diffraction measurements. For the incidence angles, $\theta_{\mathrm{i}}=20$ and $30^{\circ}$, the emerging light was recorded at $\theta_{\mathrm{r}}$, reflected white light was measured at $\theta_{\mathrm{r}}=\theta_{\mathrm{i}}=20$ and $30^{\circ}$, respectively (Fig. 4b). With increasing $\theta_{\mathrm{i}}$, reflection spectra showed a blue shift, accompanied by lowering of the optical intensity as recorded at $\theta_{0}$.

Diffraction behavior was recorded for $\theta_{\mathrm{i}}=20^{\circ}$ to $45^{\circ}$, with an increment of $5^{\circ}$ and recorded at $\theta_{\mathrm{r}}=\theta_{\mathrm{d}}$, between 28 to $60^{\circ}$, see Fig. 4c. We observed a color resolved diffraction from the CCR array hologram surface following the Bragg's law, as well as, differentiates the behavior from simple reflection law $\left(\theta_{\mathrm{i}}=\theta_{\mathrm{r}}\right)$. Diffracted color peaks also showed a blue shift from 541 to $500 \mathrm{~nm}$ as the incidence angle was moved from its minimum of $20^{\circ}$ to the maximum value of $45^{\circ}$ - optical intensity of the diffracted color light decreased as the incident angle increased. Consistent with reflection measurements, maximum peak intensity was observed at small incidence angles which systematically decreased with increasing $\theta_{\mathrm{i}}$. Incident broadband light and far-field diffracted color light with incidence angles are shown in Fig. 4d. A white-square box in this figure shows (a)

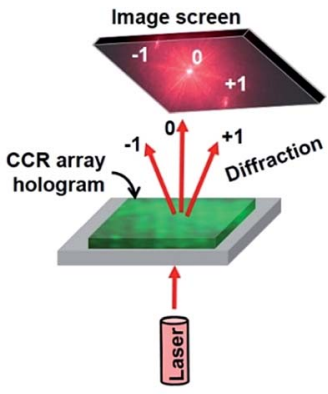

(c)

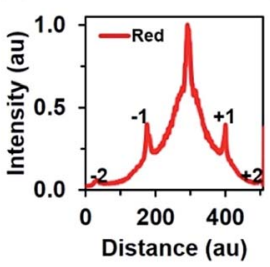

(f)

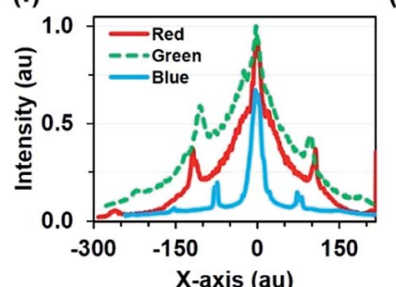

$\mathrm{X}$-axis (au) (b)

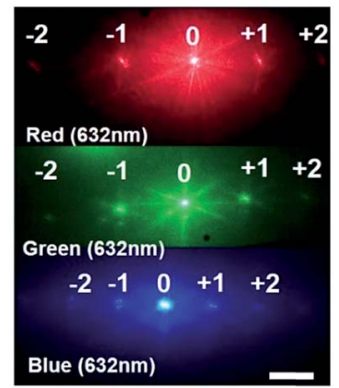

(e)

Fig. 6 (a) Conceptual diagram for the diffraction experiment. (b) Farfield diffraction patterns for red, green, and blue light normal illuminations. (c-f) Optical intensity of the diffracted red, green, and blue light along $x$-axis. (g) The DE of a CCR array hologram for monochromatic (red, green, and blue) lights illumination.

(a)
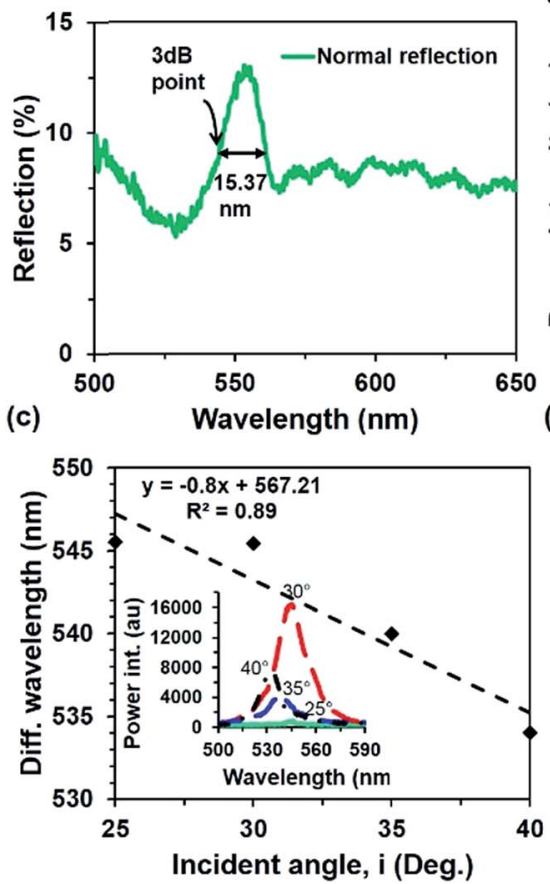

(b)
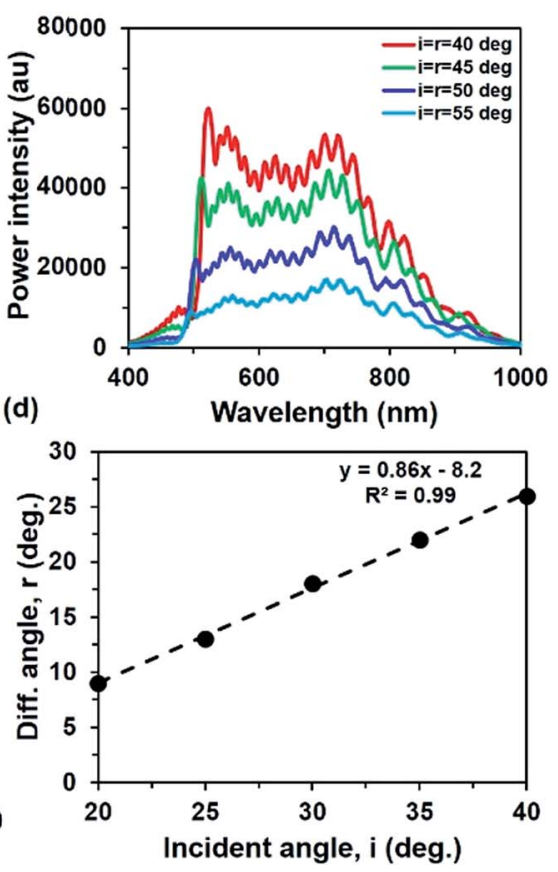

Fig. 5 Optical properties of the CCR array hologram. (a) Normal reflection from CCR array hologram surface as a function of wavelength. (B) Diffracted color light peak-wavelength as a function of incident angle. Inset shows power intensity profile with input incident angle variation. (c) Diffracted color light intensity as a function of wavelength. (d) Diffracted color light peak wavelength as a function of illumination angles. 
one example of incident broadband light, reflected white light and diffracted color (green) light at certain angle, $\theta_{\mathrm{i}}=25^{\circ}$. For $\theta_{\mathrm{r}}$ $=25,48$, and $60^{\circ}$, the far-field diffracted light showed green $(547 \mathrm{~nm})$, light-blue $(510 \mathrm{~nm})$, and dark-blue $(500 \mathrm{~nm})$ colors, respectively. We can exploit this angle resolved behavior of the CCR array hologram as a visible colorimetric based measurement systems for many practical applications.

For normal illumination of broadband light, a broadband green peak $(\lambda=554 \mathrm{~nm}, \Delta \lambda=15.37 \mathrm{~nm}$ at $3 \mathrm{~dB}$ of max) was reflected back from the CCR array holographic surface, see Fig. 5a. Normal reflection measurement was based on optical microscopic setup. Reflection spectra for normal incidence of the broadband light at CCR hologram surface was picked up by an object lens $(\times 20)$ in reflection mode. Further reflection measurements were carried out for $\theta_{\mathrm{i}}=\theta_{0}=40$ to $55^{\circ}$ with an increment of $5^{\circ}$ using the same light source (Fig. $5 \mathrm{~b}$ ). The peak wavelength of the reflected light blue-shifted as predicted by our computational modelling, as shown earlier in Fig. 3b. The peak-wavelength was plotted for diffracted color light at measurement angle, $\theta_{\mathrm{d}}=20^{\circ}$ for the different $\theta_{\mathrm{i}}=25$ to $45^{\circ}$ with increment of $5^{\circ}$, see Fig. $5 \mathrm{c}$. The diffracted peak wavelength shifted to lower values with increasing angle, due to reduced spacing parameter of multi-layer grating at the large viewing/illumination angles. Spectral recording for the diffracted color light, plotted at certain illumination angles, $\theta_{\mathrm{i}}$ is shown in inset to Fig. 5c. Angular dependence of emerging diffracted light, on different illumination angles was also studied at different spectrometer positions, confirming a linear relationship between $\theta_{\mathrm{i}}$ and $\theta_{\mathrm{d}}$, as also expected from previous results, Fig. $5 \mathrm{~d}$.

An image-screen setup was also used for far-field diffraction experiments, see Fig. 6a for setup schematics. Monochromatic light sources, red $(635 \mathrm{~nm}, 4.5 \mathrm{~mW}, \emptyset 11 \mathrm{~mm})$, green $(532 \mathrm{~nm}$, $4.5 \mathrm{~mW}, \emptyset 11 \mathrm{~mm})$ and blue (492 nm, $2.6 \mathrm{~mW}, \emptyset 11 \mathrm{~mm})$ (Thorlabs Elliptec GmbH Dortmund, Germany), were used for diffraction experiment at normal illumination, Fig. 6b-g. The diffraction distance for the 1 st and 2 nd orders from zero order increased as we moved from blue to red wavelengths in accordance with Bragg's law, $\left(\Lambda=\lambda / 2 \sin \theta_{\mathrm{i}}, \Lambda\right.$ is grating spacing, $\lambda$ is incident wavelength, and $\theta_{\mathrm{i}}$ is the incidence/ tilt angle). However, green light exhibited maximum diffraction intensity; minimum value was observed in the case of blue light. We also computed the diffraction behavior according to the experimental conditions, which confirm the
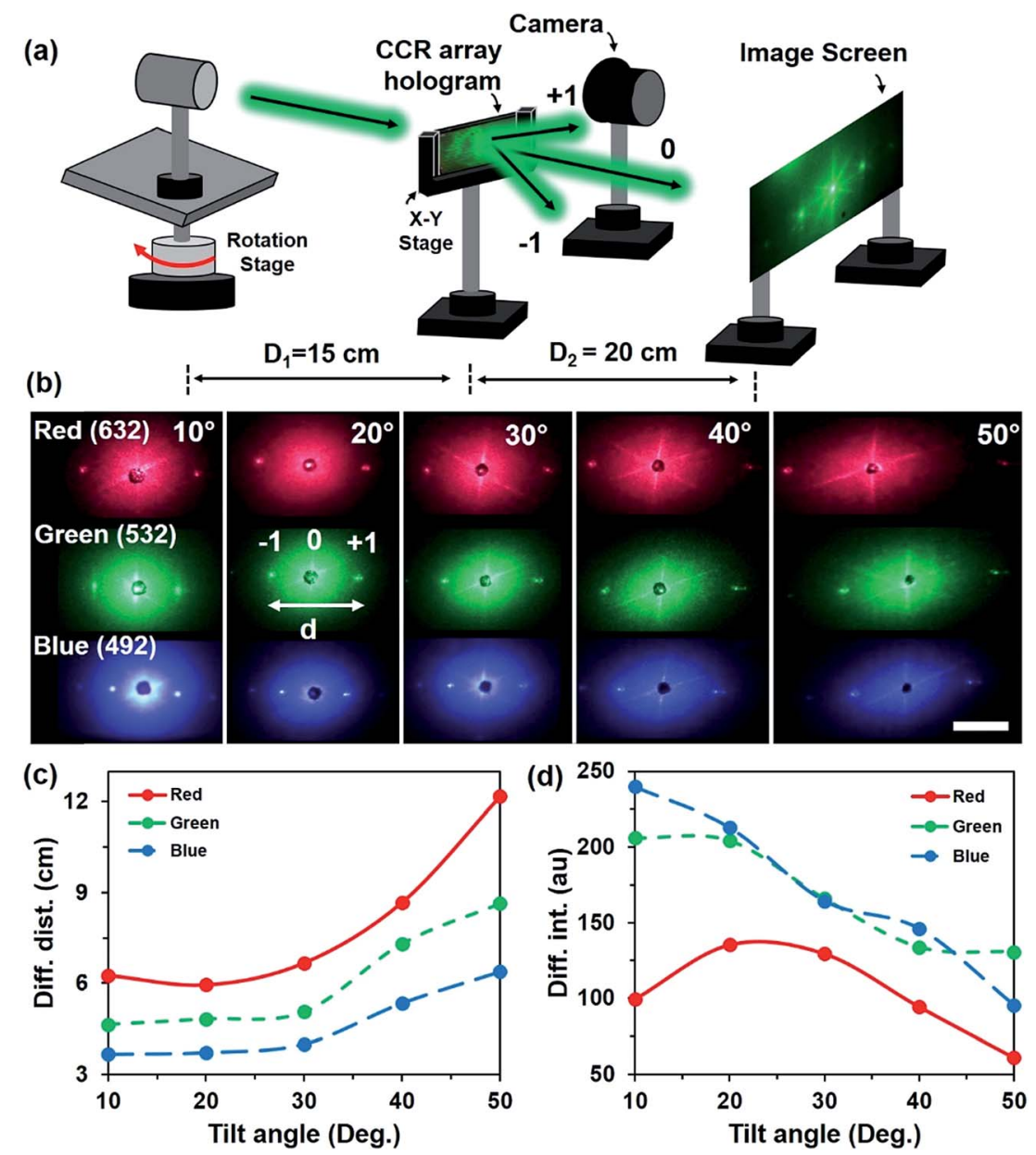

Fig. 7 Tilted directional-diffraction from flat HCCR surface. (a) Conceptual diagram for the tilted directional-diffraction experiment. (b) Far-field diffraction patterns for the red, green, and blue light through tilted illumination. (c) \& (d) Optical diffraction interspacing and intensity of the monocromatic red, green, and blue light through tilted illumination. 
experimentally recorded diffraction behavior to be consistent, Fig. 6f. Therefore, it is suggested that the diffraction properties of the CCR array hologram exhibit similar optical behavior to that of a diffraction grating. This may be due to the fact that the recorded CCR surface consisted of multi-grating structures (Fig. 1d). Furthermore, diffraction efficiencies (a ratio between intensities of diffracted and incident lights) for the 1st order for CCR array hologram were found to be maximum for green and minimum for the blue lasers, 49.8 and $16.2 \%$, respectively.

Holographic CCR array sample was also illuminated in transmission mode with three monochromatic lights (red, green, and blue) at tilt angles of $10^{\circ}<\theta_{\mathrm{i}}<50^{\circ}$, with increment of $10^{\circ}$, Fig. 7. An optical powermeter and image screen setups were used to record the information for far-field diffraction patterns at different illumination angles. Motorized precision rotation stages were used for incidence and emerging sides. The sample was positioned at a height adjustable 2D $x-y$ stage. With increasing illumination angle, the distance between diffraction spots also increases in accordance with the Bragg's law. By the same token, the red light shows maximum diffraction distance as compared with other two wavelengths. As a general trend, the diffraction intensity decreased with tilted illumination angles. However, a maximum diffraction intensity was obtained in case of green illumination, consistent with our previous results. We suggest that the diffraction intensity variation was due to the response of internal multi-grating structure ( $\mathrm{AgBr} \mathrm{NCs}$ ) of the hologram and their interaction with respect to monochromatic light.

\section{Conclusion}

The Denisyuk reflection holography technique was used to record a flat CCR hologram on a holographic polymeric film. Optical properties of the recoded hologram were characterized through reflection and diffraction experiments. Computational modeling was performed to predict angle dependent reflection, diffraction and interference behavior of the CCR array holographic surface. Image projection experiments as well simulation reveal phase-conjugated rotational properties of as made holographic devices. Under a broadband illumination, an angle dependent broadband reflection was found at the same mirror angle in the reflection plane, and narrowband color resolved diffraction at certain diffraction angles. Green light $(554 \mathrm{~nm})$ was observed to have the maximum reflection intensity in the normal reflection measurement. Diffraction angle for the color light showed a linear relationship with the incident angle of the broadband light. Second order diffraction patterns were also studied through monochromatic (red, green, and blue) light illumination - diffraction properties from the CCR array holographic surface had similarities to a diffraction grating. Monochromatic illumination also confirm the maximum DE $(\sim 50 \%)$ for the green light. Oblique illumination in the far-field directional-diffraction setting showed an increasing diffraction distance behavior with increasing illumination angle. However, the diffraction intensity generally decreased at higher tilt angles.

\section{Acknowledgements}

The authors thank Jeff Blyth for help and discussions toward this project. HB acknowledge the Leverhulme Trust for the research funding.

\section{References}

1 J. P. Hansen and S. Madhu, Appl. Opt., 1972, 11, 233-238.

2 B. Park, T. Eom and M. Chung, Appl. Opt., 1996, 35, 43724380.

3 R. A. Chipman, J. Shamir, H. J. Caulfield and Q.-B. Zhou, Appl. Opt., 1988, 27, 3203-3209.

4 H. Kim and B. Lee, Opt. Eng., 2007, 46, 094002-094014.

5 L. Zhou, J. M. Kahn and K. S. Pister, J. Microelectromech. Syst., 2003, 12, 233-242.

6 J. Hou, JOSA A, 2004, 21, 223-230.

7 H. Kim, S.-W. Min and B. Lee, Appl. Opt., 2008, 47, 64536469.

8 M. H. Bergen, J. Nichols, C. M. Collier, X. Jin, B. Raja, D. J. Roberts, P. Ruchhoeft, R. C. Willson and J. F. Holzman, Appl. Opt., 2014, 53, 3647-3655.

9 S. Wang, T. Sherlock, B. Salazar, N. Sudheendran, R. K. Manapuram, K. Kourentzi, P. Ruchhoeft, R. C. Willson and K. V. Larin, IEEE Sens. J., 2013, 13, 45344541.

10 W. Xingtao, H. Ping'an, L. Song and W. Jiyao, Geo Spatial. Inform. Sci., 2000, 3, 73-75.

11 M. S. Scholl, JOSA A, 1995, 12, 1589-1592.

12 S.-W. Park, E. Moon, H. Chung, J.-Y. Lee, C. Bae, J.-W. Kim and H. Kim, Appl. Opt., 2014, 53, 6605-6611.

13 N. S. Shaar, G. Barbastathis and C. Livermore, J. Microelectromech. Syst., 2015, 24, 1043-1051.

14 Y. Lou, H. Wang, Q. Liu, Y. Shi and S. He, Appl. Opt., 2010, 49, 5567-5574.

15 T. Sherlock, A. Nasrullah, J. Litvinov, E. Cacao, J. Knoop, S. Kemper, K. Kourentzi, A. Kar, P. Ruchhoeft and R. Willson, J. Vac. Sci. Technol., B: Nanotechnol. Microelectron.: Mater., Process., Meas., Phenom., 2011, 29, 06FA01.

16 E. Brinksmeier, R. Gläbe and C. Flucke, Prod. Eng., 2008, 2, 33-38.

17 J. Yuan, S. Chang, S. Li and Y. Zhang, Opt. Commun., 2002, 209, 75-83.

18 K. Jolic, M. Ghantasala and E. C. Harvey, J. Microelectromech. Syst., 2003, 14, 388.

19 T. Alqurashi, A. Sabouri, A. K. Yetisen and H. Butt, AIP Adv., 2017, 7, 025313.

20 T. Alqurashi, P. Penchev, A. K. Yetisen, A. Sabouri, R. M. Ameen, S. Dimov and H. Butt, $R S C A d v$., 2017, 7, 18019-18023.

21 R. Ahmed, A. K. Yetisen, S. H. Yun and H. Butt, Light: Sci. Appl., 2017, 6, e16214.

22 R. Ahmed, A. K. Yetisen, A. El Khoury and H. Butt, Nanoscale, 2017, 9, 266-276.

23 B. AlQattan, H. Butt, A. Sabouri, A. K. Yetisen, R. Ahmed and N. Mahmoodi, RSC Adv., 2016, 6, 111269-111275. 
24 J. J. Cowan, JOSA A, 1990, 7, 1529-1544.

25 R. Ahmed, A. K. Yetisen and H. Butt, ACS Nano, 2017, 11, 3155-3165.

26 H. Butt, P. R. Kidambi, B. Dlubak, Y. Montelongo, A. Palani, G. A. Amaratunga, S. Hofmann and T. D. Wilkinson, Adv. Opt. Mater., 2013, 1, 869-874.

27 A. K. Yetisen, H. Butt, T. Mikulchyk, R. Ahmed, Y. Montelongo, M. Humar, N. Jiang, S. Martin, I. Naydenova and S. H. Yun, Adv. Opt. Mater., 2016, 4, 1589-1600.
28 R. Gurney and N. Mott, Proc. R. Soc. London, Ser. A, 1938, 151-167.

29 J. D. Black and R. J. Parker, Opt. Commun., 1991, 81, 85-88.

30 D. C. O'Brien, G. E. Faulkner and D. J. Edwards, Appl. Opt., 1999, 38, 4137-4144.

31 R. Ahmed, A. A. Rifat, A. K. Yetisen, Q. Dai, S. H. Yun and H. Butt, J. Appl. Phys., 2016, 119, 113105.

32 H. Butt, A. K. Yetisen, R. Ahmed, S. H. Yun and Q. Dai, Appl. Phys. Lett., 2015, 106, 121108.

33 R. Ahmed, A. A. Rifat, A. K. Yetisen, M. S. Salem, S.-H. Yun and H. Butt, RSC Adv., 2016, 6, 56127-56133. 